\title{
Research on Algorithm of Renting Extenics Strategy Generating System
}

\author{
Jianxin $\mathrm{Li}^{1, \mathrm{a}}$ \\ ${ }^{1}$ Department of Computer Engineering, Dongguan Polytechnic, Dongguan, 523808, GuangDong, \\ China
} a⒎

Keywords: extenics; extenics transformation; strategy generation; renting

Abstract. Facing massive house rental information, the tenants consider many factors. Usually, it is difficult for them to make an optimal strategy. Considering the large amount of storage, fast computing and other characteristics, if introducing computer technology to assist in generating and evaluating strategies, it can greatly improve the level of human decision-making. Therefore, this paper explores the combination of decision science and extenics, using computer to generating strategies and evaluating strategies to improve decision-making level of decision-makers and the level of machine intelligence.

\section{Introduction}

In real life, people will encounter many contradictory problems, renting is one of the problems. When renting, you need to consider the house address, rent, area, convenience and other factors. Spending less money to rent a more suitable house is a contradictory problem. The solution of the contradiction problem depends on the generation of the strategy. But in the decision-making science, the decision-making theory rarely studies the strategy of the contradiction problem. Therefore, the strategy of the contradiction problem has always been a difficult problem for domestic and foreign scholars ${ }^{[1]}$.

International decision-making science tends to use purely mathematical ideas to solve the optimal method of choice and decision-making, the method is to build a mathematical model, based on operational research. The lack of the method is that it is difficult to establish an accurate mathematical model for practical problems (especially contradictory problems). The accuracy of the decision is unpredictable, and the credibility is low. In China, Professor Cai Wen originally establish the extenics using formal methods to deal with contradictory problems ${ }^{[2]}$. Professor Cai Wen proposes the application of divergence and convergence of diamond-like thinking to support the strategy generation. Combined with the theoretical approach, decision science and computer technology of the extenics project ${ }^{[3]}$, we can design the Extenics Strategy Generation System $(\mathrm{ESGS})^{[4]}$ to generate a solution to the problem.

According to the theory of ESGS, this paper takes the contradiction problem of renting as the application example, establishes the solution model from the condition to the target, and tries to make better decision by balancing the influence factors (constraint) through certain transformation or expansion method. This paper uses better strategies to solve the problem of contradictions, to improve the decision-makers, and to improve the level of decision-making and the level of machine intelligence.

General steps for the extenics strategy generation method are as follows: (1)Define the goal and condition of the problem by using the method of the contradiction problem, and use the primitive 
representation; establish the extenics model of the problem and the extenics model of its nuclear problem.(2) Establish the contradictory problem of the decision function--compatibility function. In the extenics mathematics, it uses the associated function to describe the certain degree of nature of the elements of the domain. (3)Base on the expansion of the basic element analysis method, to analysis the problem of expansion, as well as the expansion of the object for the expansion of the operation.(4)According to the evaluation of excellentness, the generated extenics strategy is selected.

Section 1 describes the architecture of the rental system; Section 2 describes the design and implementation of several core algorithms; Section 3 describes the results of the system operation; finally, a conclusion is given.

\section{Construction of Renting Extenics Strategy Generating System}

According to the general steps of the extenics strategy generation method, we can design the rental strategy generation system as shown in Fig. 1. It is easy to see from the figure that the system adopts three layers of $\mathrm{C} / \mathrm{S}$ architecture. It includes several modules mainly: user interface, problem analysis and modeling, extenics transformation, evaluation promotion and background ontology knowledge management. The function is used to define the incompatibility problem. The problem is modeled as an incompatible problem. The extenics model is used to solve the contradictory problem. The problem is to solve the problem of contradiction. The method of providing the method is used to evaluate the extenics strategy, so that the user adopts the rental strategy. The background ontology knowledge management is used to maintain the ontology knowledge of the house information and membership information.

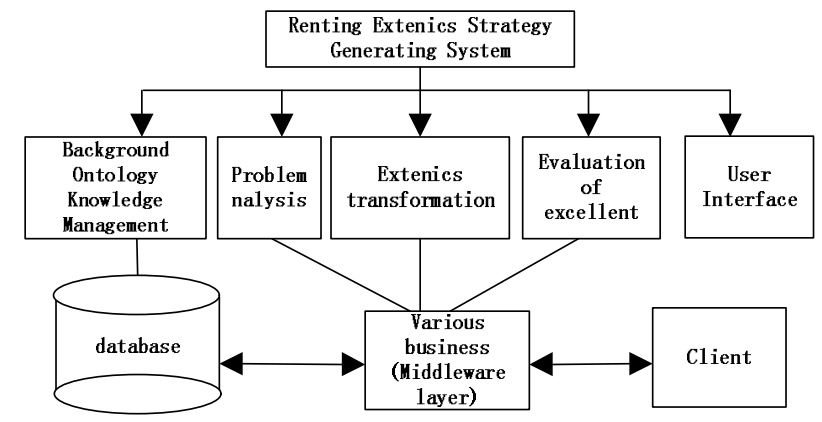

Fig. 1 rental strategy generation system architecture

\section{Design and Implementation of Core Algorithm}

The system has the following three core algorithms: extenics transformation algorithm, evaluation algorithm, crawler algorithm. The following are given a brief description.

\section{Extenics transformation algorithm}

The efficiency of the extenics transform algorithm directly affects the efficiency of the whole system, so it is very important to construct an efficient extenics transformation algorithm, and reducing the number of database access is the most effective way to improve the efficiency of the system.

In this system, the main operating object the "house information" is ontology knowledge, it exists in the form of data records in the database table. In the process of extenics transformation, we need to access these house data records. To improve efficiency, we use caching technology to access 
the house table once in a logical operation (extenics transformation). Therefore, we design the extenics transformation algorithm as shown in the following flow figure (Fig. 2) for the system.

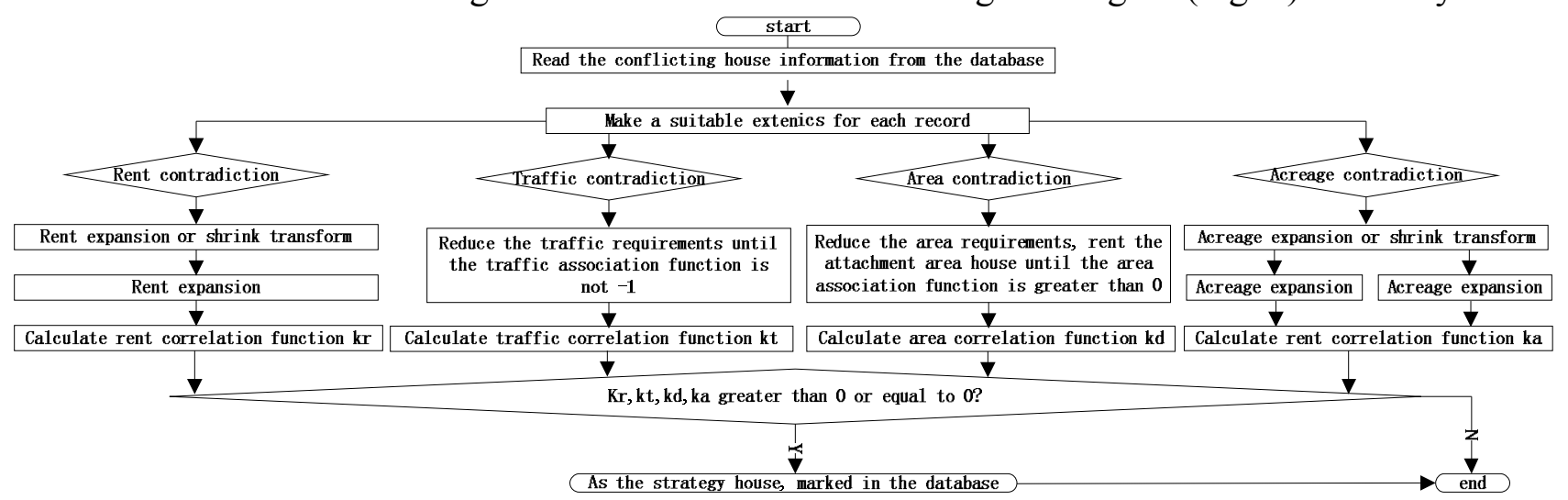

Fig. 2 renting extenics transform flow

\section{evaluation algorithm}

According to the literature [3], strategy $\mathrm{Z}$ generated by the system is the measure of the value $\mathrm{SI}=\{$ area,rent,acreage,traffic $\}$. The normative degree of correlation about $Z$ is $k_{i}=\frac{K(Z)}{\max |K(x)|}(i=1,2, \ldots, n) . \mathrm{N}$ is the number of strategies. The acreage, the rent, the area and the traffic convenience corresponding to the weight coefficient are $a_{1}, a_{2}, a_{3}, a_{4} \quad\left(0 \leq a_{1}, a_{2}, a_{3}, a_{4} \leq 1\right)$. Then the better degree of the house strategy is $C(Z)=\sum_{i=1}^{n} a_{i} k_{i}$.

\section{reptile program}

The system uses HttpClient ${ }^{[6]}$ technology to achieve the reptile program. HttpClient is a subproject of Apache Jakarta Common which provides a client programming toolkit and supports the HTTP protocol. Using HttpClient to access the network page information and coexisting in the database algorithm flow are as shown below Fig. 3.

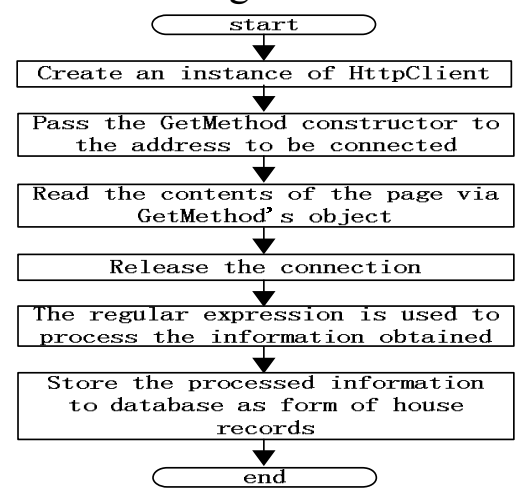

Fig. 3 Get the page information flow through the crawler program

\section{Experimental results and analysis}

By coding to Implement the system, we get main page of renting extenics strategy generation system, users can choose the favorite area, budget rent, the desired area and convenience of 
transportation. For example, the budget of 700 yuan in Tianhe District, rent 20 square meters, convenient transportation facilities. After the submission, the analysis determines whether the problem is contradictory. Visible strategy library which has a user requirements of the house, users can click the record to get the house details directly.

At the same time, if users think that the optional strategy is less, you can also click on the extenics transform button to get more suitable house. After the extenics of the transformation, the strategy houses change from one to 26 .

Users is likely to be more difficult in the 26 house strategy to select the most suitable for their own house in a short time, instead, the user can enter the area, rent, area and convenience of traffic factors, excellent evaluation, to find The most suitable for their own house.

\section{Conclusions}

Based on the theory of decision-making science, extenics science and computer science, this topic constructs the system of renting extenics strategy, so as to explore the application of decision-making science and extenics science on computer, try to carry out the strategy Automated generation and evaluation to achieve decision makers' level of decision-making and the goal of improving machine intelligence.

On the other hand, the system has a great application value. It can also produce social benefits. At present, the test data (house information) that the system uses are from the network. If the members open rental housing release channel (the function has been implemented in the system but not open), or through other legitimate channels to obtain accurate and reliable house information, this system will provide people a renting information platform. The renting users will be able to make the best decision, but also accurately and quickly find a job to meet the needs and housing requirements. It will break the current renting model that people rent a house through the intermediary. And it will be our next step to do the work.

\section{References}

[1] Chen Julong,Cong Lin, Strategy-generated extenics method. Journal of Guangdong University of Technology,2001.18(1):84-87.

[2] Cai Wen. Extenics theory and its application[J]. Chinese Science Bulletin , 1999,44(17):1538-1548.

[3] Yang Chongyan, Cai Wen. Extenics engineering [M]. BeiJing: Science Press, 2007.1-344.

[4] Li luxi, Yang Chongyan, Li Huawen. Extenics strategy generation system [M].BeiJing:Science Press, 2006:1-232.

[5] Li Chenxiao, Li Weihua, Zou Zhangzhi. Research on Extenics Optimization Algorithm of Tourism Strategy Generation System [C]// Progress in Chinese Artificial Intelligence(2009). BeiJing,2009:633-637.

[6] Apache Software Foundation. Jakarta Commons HttpClient[EB/OL].

[7] http://hc.apache.org/httpclient-3.x/:2001-2008. 